\title{
Increased expression of metastasis-related genes in hypoxic cells sorted from cervical and lymph nodal xenograft tumors
}

\author{
Naz Chaudary ${ }^{1,2}$ and Richard P Hill ${ }^{1,2,3}$
}

Solid tumors contain regions of poor oxygenation that relate to the abnormal vascular network. Clinical investigations in cervical carcinoma have shown that positive lymph node status in patients with cervical carcinoma correlates with hypoxia. Earlier, in an orthotopic cervical cancer model, we had shown that exposure to acute hypoxia enhances lymph node metastasis. This study describes a technique for sorting hypoxic cells directly from the cervical xenograft model and reports the expression of 'metastasis-related' genes in hypoxic cells from xenografted cervix and lymph node tumors. Tumor cells were sorted on the basis of DsRed fluorescence and the sub-population of hypoxic cells was sorted on the basis of carbonic anhydrase-9 (CA-9) expression. Quantitative RT-PCR was conducted to measure changes in gene expression in the hypoxic cells sorted from primary cervix tumors and lymph node metastases. Immunohistochemistry was used to track changes in protein expression in sections of the same tumors. Metastasis-related genes, CXCR4, UPAR, VEGFC, Hdm2, and OPN, were observed to be upregulated at gene and protein levels in the primary tumors and nodal metastasis from the orthotopic transplants. In particular, the hypoxic cells sorted from orthotopically transplanted cervix tumors and their lymph node metastases from mice exposed to cyclic (intermittent) hypoxia showed higher levels of expression of these genes. These results are consistent with the hypothesis that these genes may be involved in regulating lymph node metastasis in cervical cancers under hypoxic conditions and provide support to the concept cyclic hypoxia that plays an important role in this process. Our methodological study emphasizes the technique of cell sorting to identify hypoxic cells using CA-9, which may aid in improving prognostic capabilities and in designing rational therapeutic strategies by focusing on hypoxia-specific gene expression profiles of patients. The technique can be applied to identify other potential 'hypoxia-related' genes of interest for tumor growth and metastasis.

Laboratory Investigation (2009) 89, 587-596; doi:10.1038/labinvest.2009.16; published online 23 March 2009

KEYWORDS: CA-9; cell sorting; cervical carcinoma; gene expression; hypoxia; lymph node metastasis

The heterogeneous microenvironment of solid tumors contains regions of high acidity, low nutrient availability, and poor oxygenation (hypoxia), which relate to the abnormal vascular network that exists in tumors. ${ }^{1-3}$ This abnormal vasculature leads to areas of both chronic and acute (cyclic) hypoxia because of intermittent blood flow. When the demand for molecular oxygen that is necessary for cellular function exceeds the vascular supply, cellular hypoxia occurs. Genetic and adaptive changes in cancer cells, likely required for further survival and proliferation, are essential for tumor progression, and may occur at an increased frequency because of genomic instability. Some cells may adapt to the environmental stress, escape necrosis and apoptosis and, as a result, may develop a more aggressive phenotype. ${ }^{4,5}$ Specifically, hypoxia has been proposed to be a key microenvironmental factor that can alter the expression of a myriad of genes, including transcription factors, growth factors, cytokines, and DNA repair enzymes. It is a mechanism by which tumor cells can acquire genomic instability, and most tumor cells possess a significant number of genomic changes. Furthermore, during hypoxia, an intricate balance exists between factors that induce or counteract apoptosis, or even stimulate proliferation. ${ }^{4,6-13}$

A hypoxic microenvironment in solid tumors seems to contribute to metastatic progression. ${ }^{14-18}$ Cervical carcinoma

\footnotetext{
'Division of Applied Molecular Oncology, Ontario Cancer Institute/Princess Margaret Hospital, Toronto, ON, Canada; ${ }^{2}$ Department of Medical Biophysics, University of Toronto, Toronto, ON, Canada and ${ }^{3}$ Department of Radiation Oncology, University of Toronto, Toronto, ON, Canada

Correspondence: Dr RP Hill, PhD, Department of Medical Biophysics, University of Toronto, Ontario Cancer Institute, Princess Margaret Hospital, 610 University Avenue, Rm 10-113, Toronto, ON, Canada M5G 2 M9.

E-mail: hill@uhnres.utoronto.ca

Received 16 October 2008; revised 22 December 2008; accepted 2 January 2009
} 
spreading to the pelvic and aortic lymph nodes is a common occurrence, and is one of the primary determinants of outcome for patients. Pitson et al, ${ }^{19}$ showed a significantly increased risk of nodal or distant metastases in patients with hypoxic cervix carcinomas, independent of tumor size, thereby suggesting that tumor hypoxia is a prognostic factor associated with selection for a metastatic phenotype. Our earlier work has shown that the level of hypoxia in the primary tumor predicts the likelihood of lymph node metastasis in patients. ${ }^{20}$ Furthermore, using a strategy designed to simulate acute (cyclic) hypoxia, we have shown that, in orthotopic cervical xenografts intermittent hypoxia treatment accelerates lymph node metastasis. ${ }^{21}$ There is significant heterogeneity in tumor oxygenation between individual tumors, ${ }^{22}$ and recently, Bachtiary $e t a^{23}$ have shown that genes can be expressed quite variably within a single cancer. Furthermore, both acute and chronic hypoxia can occur variably in tumors, and the relative importance of these different modes of hypoxia for metastatic spread is currently under investigation. ${ }^{24}$

Solid tumors are composed of multiple sub-population of cells (e.g. tumor cells and non-tumor cells) and as such the analysis of gene expression may not be representative of the tumor cells. ${ }^{25}$ Tumor progression has been recognized as the product of an evolving crosstalk between different cell types with the tumor and its surrounding supporting tissue or tumor stroma. The relative amount of stroma and its composition vary considerably from tumor to tumor. Interactive signaling between tumor and stroma contributes to the formation of a complex multicellular organ. Cancer cells themselves can alter their adjacent stroma to form a permissive and supportive environment for tumor progression. ${ }^{26}$ Recent cell separation techniques have provided many advantages for studying cell populations prepared from solid tumors. ${ }^{27-30}$ However, current methods used to detect hypoxic cells are often technically complex, invasive, or require administration of chemicals to mark hypoxic cells. We have adapted the earlier work of Olive et $a l^{27}$ and Vordermark et $a l^{31}$ to devise a simple separation method for hypoxic cells on the basis of carbonic anhydrase-9 (CA-9) expression in an orthotopic xenograft cervical model. ${ }^{32}$ CA-9 is a well-known marker of tumor hypoxia identifiable by its intense surface membrane staining, commonly seen in hypoxic tissue. ${ }^{33-35}$ Recently, Iakovlev et $a l^{36}$ showed that CA-9 labeling was correlated with direct $\mathrm{pO}_{2}$ measurements in cervical carcinoma and that there was significant intratumoral heterogeneity. Our study further supports CA-9 as a suitable marker for identifying hypoxia and presents findings showing upregulated expression of a series of metastasis-related genes in sorted CA-9-positive (hypoxic) cells. These genes that are identified from the literature are upregulated in vivo, particularly in the hypoxic cells in the primary tumors and their lymph node metastasis from animals exposed to the intermittent (cyclic) hypoxia regime.

\section{MATERIALS AND METHODS \\ Cell Culture}

ME180, human cervical carcinoma cells, stably transfected and labeled with DsRed, were cultured as described earlier. ${ }^{21}$ Briefly, the cells were grown in vitro as monolayers in $\alpha$-minimal essential medium ( $\alpha$-MEM) (Gibco BRL, Burlington, ON, Canada) supplemented with penicillin/ streptomycin and 10\% fetal bovine serum (FBS; Wisent, St Bruno, QC, Canada). The cells were grown under selection pressure with $400 \mu \mathrm{g} / \mathrm{ml}$ G418 (Life Technologies, Inc., Burlington, ON, Canada) and incubated in $5 \% \mathrm{CO}_{2}$ and humidified air at $37^{\circ} \mathrm{C}$.

\section{Orthotopic Cervical Implantation}

The tumor cells were implanted orthotopically into the cervix of SCID mice as described earlier. ${ }^{32}$ All animal experiments were carried out according to protocols approved under regulations of the Canadian Council on Animal Care.

\section{In Vivo Cyclic Hypoxia}

Cyclic hypoxia treatment in vivo was performed as described earlier. ${ }^{21}$ Briefly, the hypoxia treatment consisted of 12 cycles of $10 \mathrm{~min}$ in $7 \% \mathrm{O}_{2}$, and balance $\mathrm{N}_{2}$ followed by $10 \mathrm{~min}$ in air, given $4 \mathrm{~h} /$ day/week ( 7 days) for 3 weeks during tumor growth. Normoxic animals were handled similarly but subjected to air breathing only. The mice were killed immediately after the last (hypoxic) cycle and the primary cervix and lymph node metastatic tumors were immediately removed and flash frozen in OCT for immunohistochemistry, immunofluorescence, and separately for RNA extraction. We have earlier shown that cyclic hypoxia causes a decrease in tumor growth relative to air-treated mice. ${ }^{21}$ At harvest, the tumor sizes are $\sim 0.25 \mathrm{~g}$.

\section{Single-Cell Suspensions and Cell Fluorescence Activated Cell Sorting Analysis}

Single-cell suspensions from pooled primary cervix and metastatic lymph node tumors, were prepared by an enzymatic technique adapted from Siemann and colleagues. ${ }^{28,29}$ Briefly, tumors (primary cervix and lymph node) were removed from the mice, minced finely, and added to an enzyme cocktail mix consisting of $0.025 \%$ collagenase (SigmaAldrich, Oakville, ON, Canada), 0.04\% DNAse (Sigma), and $0.05 \%$ pronase (Sigma). The enzyme preparations $(35 \mathrm{ml}$ enzyme solution per gram of tissue) were incubated for $1 \mathrm{~h}$ with constant agitation on a rotating wheel at $37^{\circ} \mathrm{C}$. After incubation, the tumor cell suspensions were passed through 40- $\mu \mathrm{m}$-pore nylon mesh filters into $10 \%$ FBS and centrifuged at $1000 \mathrm{rpm}$ for $7 \mathrm{~min}$ at $4^{\circ} \mathrm{C}$. The single-cell suspensions were resuspended in complete $\alpha$-MEM (with 10\% FBS). All procedures, except incubation, were performed at $4^{\circ} \mathrm{C}$. Cell number and viability were measured using a hemocytometer and vital-dye trypan-blue, respectively.

Pelleted cells were resuspended in a primary human antiCA-9 (Novus Biologicals) MN75 monoclonal antibody 
$\left(2.5 \mu \mathrm{l} \mathrm{M} 75 / 5 \times 10^{5}\right.$ cells) and incubated for $15 \mathrm{~min}$ at room temperature. The cells were rinsed twice with $4 \%$ FBS in PBS and then centrifuged for $5 \mathrm{~min}$ at $1000 \mathrm{rpm}$. The cells were resuspended with secondary goat-anti-mouse FITC (green fluorescence) antibody ( $1 \mu \mathrm{l} / 5 \times 10^{5}$ cells) and incubated for $15 \mathrm{~min}$ at RT in the dark. The cells were washed once with $4 \% \mathrm{FBS}$ in PBS and centrifuged for $5 \mathrm{~min}$ at $1000 \mathrm{rpm}$. They were resuspended in $4 \% \mathrm{FBS}$ in PBS $\left(0.5 \mathrm{ml}\right.$ for $5 \times 10^{5}$ cells). Hoechst $33342(10 \mu \mathrm{M})$ was added as a control to assess the population of viable tumor cells, and incubated for $15 \mathrm{~min}$ at $37^{\circ} \mathrm{C}$. Hoechst 33342 was not included in the sorted samples, other than to help define gates on the basis of forward and side scatter. All samples were filtered through $40-\mu \mathrm{m}$-pore nylon mesh into $0.5 \mathrm{ml}$ of $4 \%$ FBS in PBS. From the DsRedsorted tumor cells, hypoxic cells were sorted on the basis of anti-MN75 monoclonal human antibody positivity (initial gates were set using forward and side scatter and Hoechst 33342 to identify the tumor (DsRed) cell population) (Supplementary Figures 1 and 2). Flow cytometry was conducted using MoFlo (Dako) in the PMH Flow Cytometry Facility. Sorted cells $\left(10^{6}\right.$ cells from primary cervix tumor and $10^{4}$ from lymph node metastasis; from one experiment of 25 pooled mice) were pelleted and flash frozen for later extraction of RNA.

\section{Flow Cytometry Analysis In Vitro and In Vivo}

ME180 DsRed cells were treated with cyclic hypoxia in vitro for $24 \mathrm{~h}\left(7 \% \mathrm{O}_{2}\right.$ for $30 \mathrm{~min}$ or $0 \% \mathrm{O}_{2}$ for $\left.30 \mathrm{~min}\right)$ after which the cells were labeled with the primary antibodies noted for immunofluorescence. Secondary antibodies of Allophycocyanin (APC) donkey anti-goat and goat-anti-rabbit (Alex Fluor 680; Invitrogen Molecular Probes) were used to identify the target genes (FL4 channel-red), and goat-anti-mouse FITC was used to identify the CA-9-positive cells in the FL1 channel (green). Double positive cells for CA-9 and target genes were quantified after gating on the side and forward scatter and cell viability using 7-AAD (7-amino-actinomycin D). A similar procedure was used to isolate double positives and correlation of target genes in the primary tumor. Initially, CA-9 and DsRed positives were sorted as described above and these cells were then used to stain for the target genes of interest.

\section{RNA Extraction and Real-Time Quantitative PCR Assay}

Total RNA was extracted, using the Qiagen RNeasy Mini Extraction kit (Qiagen, Mississauga) from frozen tissue or cultured ME180 cells, and frozen cell pellets, according to the manufacturer's instructions. From 0.5 to $1 \mu \mathrm{g}$ of DNasetreated total RNA, first-strand cDNA was reverse-transcribed using OmniScript (Qiagen) according to the manufacturer's instructions. For real-time PCR detection, $1 / 10$ of the cDNA was mixed with primers $(0.3 \mu \mathrm{M}$ final concentration), $\mathrm{ddH}_{2} \mathrm{O}$, and SYBER Green Master Mix (Applied Biosystems, Foster City, CA, USA) for PCR amplification with a final well volume of $20 \mu \mathrm{l}$. Primers for the genes of interest were designed using the Primer Express software program (PE; Applied Biosystems) and synthesized by Invitrogen (Supplementary Table 1 for primer sequences). The real-time PCR protocol was $50^{\circ} \mathrm{C}$ for $2 \mathrm{~min}$ (pUNG deactivation to prevent carry-over contamination), $95^{\circ} \mathrm{C}$ for $10 \mathrm{~min}$ (hot start), plus 40 cycles of $95^{\circ} \mathrm{C}$ for $15 \mathrm{~s}$ (melting), and $60^{\circ} \mathrm{C}$ for 1 min (annealing and extension), as recommended by an ABI manufacturer. The reactions were run and analyzed with an ABI PRISM 7900 Sequence Detector (Applied Biosystems). Human L32, a ribosomal protein, was used as an endogenous control to normalize the results. Levels of L32 did not differ significantly from normoxia to hypoxia. Samples were run in duplicate or triplicate to obtain the corresponding threshold cycle $\left(C_{t}\right)$, values which were used as a direct quantitative measurement of the gene expression level and expressed as mean ( \pm s.d. or s.e.). Fold differences of hypoxia/normoxia were calculated. Initial experiments included using universal human RNA to ensure that no variability was observed with the L32 control. VEGFA was used as a positive control for hypoxic treatment. Negative controls were without cDNA templates.

\section{Immunohistochemical Analysis}

Immunohistochemical staining was performed by the Pathology Research Program at Toronto General Hospital and at the Drug Development Laboratory at OCI/PMH. Frozen sections of primary cervix and lymph node tumors $(5 \mu \mathrm{m})$ were air-dried and fixed in cold acetone or $2 \%$ paraformaldehyde for $10 \mathrm{~min}$. The sections were then air-dried and washed in PBS. Endogenous peroxidase was blocked with $0.3 \%$ hydrogen peroxide and the slides were washed in water. An avidin/biotin blocking kit (Vector Labs) was used to block avidin/biotin activity within the tissue for $15 \mathrm{~min}$. The slides were washed with PBS. Sections were incubated with a normal serum protein block (Vector Labs), then drained and incubated with a primary antibody at room temperature in a moist chamber (see Supplementary Table 2 for antibody conditions). The slides were washed with PBS and incubated with a biotinylated secondary antibody for $30 \mathrm{~min}$ (Vector Labs), washed with PBS again, and then incubated with a peroxidase-conjugated ultrastreptavidin labeling reagent (Vector Labs). Color development was carried out with freshly prepared Nova Red (Vector Labs). Finally, the sections were counterstained with Mayer's hematoxylin and mounted in Permount (Fisher). Whole slide images were scanned (at $\times 20$ magnification) in the Advanced Optical Microscopy Facility at $\mathrm{OCI} / \mathrm{PMH}$, and the Positive Pixel Count Algorithm in the ImageScope software (Aperio Technologies) was used to quantify the positive signal.

\section{Immunofluoresence Dual Labeling of CA-9 and Target Genes}

Frozen sections of primary cervix tumors and lymph node metastasis were cut at 5- $\mu \mathrm{m}$ thickness on a Leica CM1950 Cryostat. The slides were fixed in $2 \%$ formaldehyde/PBS for 
$20 \mathrm{~min}$ at $4^{\circ} \mathrm{C}$ and rinsed in PBS. Primary antibody incubation with target markers and CA-9 cocktail was carried out for $16 \mathrm{~h}$ at RT using CXCR4 (Santa Cruz goat polyclonal, 1:1000 dilution); uPAR (Chemicon goat polyclonal, 1:25 dilution); OPN (R\&D goat polyclonal, 1:200 dilution); Mdm2 (R\&D rabbit polyclonal, 1:500 dilution); VEGFC (Zymed rabbit polyclonal, 1:300 dilution); and CA-9 (Novus Biologicals; mouse monoclonal MN75, 1:200 dilution). Negative controls were incubated in PBS only. Cy3/Cy5conjugated secondaries were incubated for $60 \mathrm{~min}$ (Anti-mouse IgG Jackson ImmunoResearch, Cy3- or Cy5conjugated, 1:200; anti-rabbit IgG Jackson ImmunoResearch, Cy3-conjugated, 1:200; anti-goat IgG. Jackson ImmunoResearch, Cy3-conjugated, 1:200.) Nuclei were labeled in a DAPI solution for $5 \mathrm{~min}$, rinsed in PBS, and blow-dried.

Immunofluorescence analysis of the images was carried out with the Olympus BX50 with a $100 \mathrm{~W}$ mercury arc burner, a $20 \times$ UPlanFL NA 0.75 object (Olympus, Canada). Fluorescent cubes of \#31000 for DAPI, \#31002 for Cy3, and \#41008 for Cy5 were used (Chroma, Rockingham, VT, USA). Images were tiled using the software MCID (MicroComputer Imaging Device) version Elite 7.01 .0 build 207, (Imaging Research St Catharines, Canada) which controlled both the Quantix Cooled CCD camera (Photometrics, Tucson, AZ, USA) and the Ludl Precision stage with a MAC5000 controller (Ludl Electronic Products, Hawthorne, NY, USA). The images were analyzed using MCID Analysis (Interfocus Imaging Ltd., UK). Flat-field correction was performed for each fluorochrome using a blank slide before acquisition. This correction was then applied to the appropriate field of view by the MCID software while tiling sequential fields.

\section{Statistical Analysis}

One-way analysis of variance followed by the Newman-Keuls post-test, using Graphpad software, was used to determine the significance between normoxic and hypoxic treatment groups, as well as between primary cervix and lymph node groups. Statistical significance was assumed at $P$-values of $<0.05$.

\section{RESULTS}

\section{Sorting and Gene Expression Profile after Acute Cyclic Hypoxia in the Orthotopic Cervical Model in Sorted and Unsorted Tumor Cells}

We initially verified that the expression of the metastasisassociated genes in ME180 cells (Supplementary Table 3) was affected by exposure to chronic hypoxia $\left(0.2 \% \mathrm{O}_{2}\right.$ for $\left.24 \mathrm{~h}\right)$ in vitro. Most genes were highly upregulated by $24 \mathrm{~h}$ of chronic hypoxic exposure $\left(0.2 \% \mathrm{O}_{2}\right)$ followed by $1 \mathrm{~h}$ of reoxygenation in relation to normoxia. Furthermore, the expression levels returned to normoxic levels after $18 \mathrm{~h}$ of reoxygenation, indicating that the gene expression changes were transient (Supplementary Figure 3).

The profiles and gates used for sorting CA-9 positive and negative cells populations from primary cervix tumors and lymph node metastasis in normoxia (air)-treated mice, are illustrated in Figure 1a. Only 20 and $10 \%$ (s.d. \pm 1 and $0.5 \%$, respectively) of cells sorted from the primary cervix tumor and lymph node metastasis, respectively, were CA-9 positive in the cell population derived from tumors exposed to normoxia. These values are averaged from four individual sorting experiments. Approximately $10-15 \%$ of the cells in the profile did not express DsRed (seen in the lower area of the sorting profile). These cells most probably represent normal cells, although there may also be some tumor cells that have lost expression of DsRed. The profiles and gates used for sorting CA-9 positive and negative cells from primary cervix tumors and lymph node metastasis in cyclic hypoxic-treated mice (exposed to 12 cycles of $10 \mathrm{~min}$ in $7 \%$ $\mathrm{O}_{2}$, balance $\mathrm{N}_{2}$ for $10 \mathrm{~min}$ in air, given $4 \mathrm{~h} /$ day for 3 weeks during their growth) are illustrated in Figure 1b. Approximately 80 and $60 \%$ (s.d. \pm 5 and $2 \%$, respectively) of the cells sorted from these primary cervix tumor and lymph node metastasis, respectively, were CA-9 positive.

We initially examined the gene expression levels in the pooled unsorted (whole tumor tissue) primary cervical tumors and lymph node metastatic tumors exposed to cyclic hypoxia during their growth. A small increase was observed in the expression of a majority of the studied genes relative to tumors that were not exposed to cyclic hypoxia (normoxia) (Figure 2a). Of the 11 genes tested, 8 were significantly upregulated in the primary tumors and 7 of these genes were upregulated in the lymph node metastasis. As the total tumor cell population may not represent gene expression in the hypoxic tumor cells, we examined gene expression specifically in the (sorted) hypoxic (CA-9 positive) population of tumor cells from primary cervix and lymph node metastatic tumors exposed to normoxia or cyclic hypoxia. The hypoxic cells were sorted on the basis of CA-9 expression from the tumor cell population (DsRed expression), and the gene expression in these cells was analyzed relative to cells not expressing CA9 from the same tumors. Interestingly, there was limited upregulation of these genes in CA-9-positive tumor cells from tumors exposed to normoxia (Figure 2b); however, there were large increases in gene expression in the CA-9-positive tumor cells from the tumors exposed to cyclic hypoxia (Figure 2c). Ten genes (including CA-9) were upregulated in the primary cervix tumors and in lymph node metastasis excluding IL-8. In the ME180 primary cervix tumors, CXCR4 and $\mathrm{Hdm} 2$ and in the lymph node metastases, uPAR, VEGFC, and OPN showed the highest upregulation.

\section{Immunolocalization Analysis of Upregulated Genes Under Cyclic Hypoxia}

We next examined whether the changes in mRNA reflected the changes in protein expression. We focused our attention on the expression of the six genes, CXCR4, VEGFC, Hdm2, OPN, uPAR plus CA-9, which showed the greatest increase in expression after cyclic hypoxia in primary and lymph node 

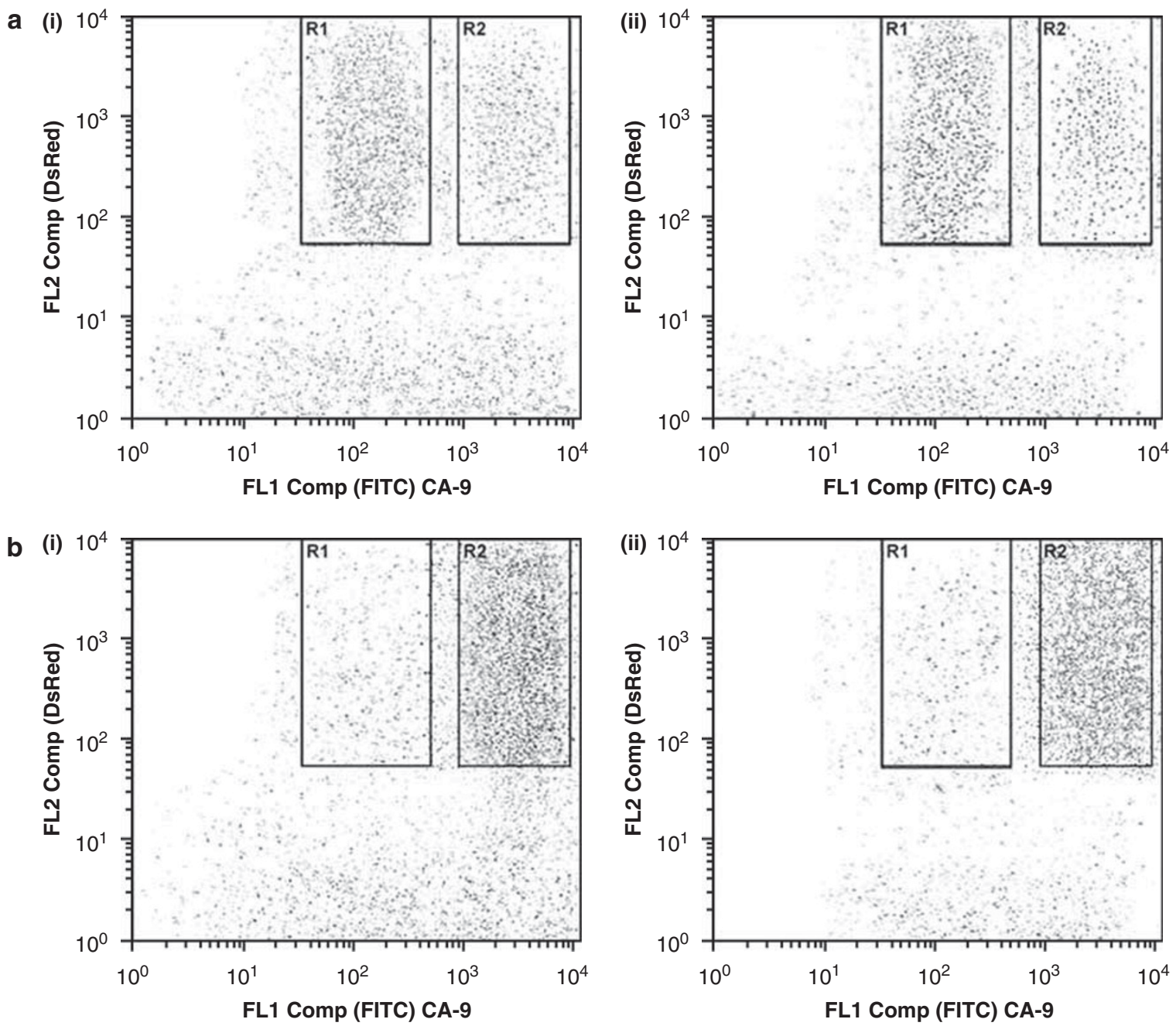

Figure 1 Cell-sorting profiles from the primary cervix tumor and lymph node metastasis after normoxia and cyclic hypoxia. (a) Cell sorting of CA-9 negative (R1) and positive (R2) populations from normoxia-treated mice (i) primary cervix tumors and (ii) lymph node metastasis. Cells were gated on FL2 (DsRed) and FL1 (FITC). (b) Cell sorting of CA-9 negative (R1) and positive (R2) populations from hypoxic-treated mice (i) primary cervix tumors and (ii) lymph node metastasis. Cells were gated on FL2 (DsRed) and FL1 (FITC). Refer to Materials and Methods for experiment details.

tumors. Immunohistochemistry was conducted to analyze the expression patterns after cyclic hypoxia exposure in vivo (Figure 3a). In the tumor cells in both the primary cervix and the lymph node tumors, the localization pattern of CXCR4 showed positive membrane staining with cytoplasmic localization of the receptor, ${ }^{37}$ and VEGFC showed a positive membrane stain. ${ }^{38,39}$ uPAR staining was observed to be localized in the cytoplasm, but specific regions showed membrane staining consistent with literature reports. ${ }^{40}$ $\mathrm{Hdm} 2$ stained positive in both the nucleus and cytoplasm. The staining of OPN appeared in the cytoplasm with slight membrane staining of tumor cells in both the primary cervix and the lymph node tumors. In addition, CA-9 showed the presence of hypoxic regions in the ME180 xenografts. The localization patterns of CA-9 revealed abundant staining in cyclic hypoxia-treated mice $(80 \%$ in the primary cervix and $60 \%$ in the nodes) compared with normoxia-treated mice
(20\% in the primary cervix and $10 \%$ in the nodes), consistent with the fact that these mice were killed immediately after exposure to the cyclic hypoxia-breathing regime and consistent with the results from the sorting experiments. All the six genes analyzed were more abundantly expressed in tumors derived from mice exposed to cyclic hypoxia compared with tumors from normoxic mice. These differences in protein expression levels were quantified using the Positive Pixel Count Algorithm/Image Scope (Aperio), which confirmed significantly increased levels of these genes in the tumors exposed to cyclic hypoxia (Figure 3b). The quantification of the positive localization signal is representative of tumor cells.

Immunofluoresence analysis showed strong-to-moderate correlation of CA- 9 with the target genes of interest in both the primary tumor and lymph node metastasis (Figure 4a) indicating a $>80 \%$ correlation (CXCR4, uPAR, and OPN) of 
a
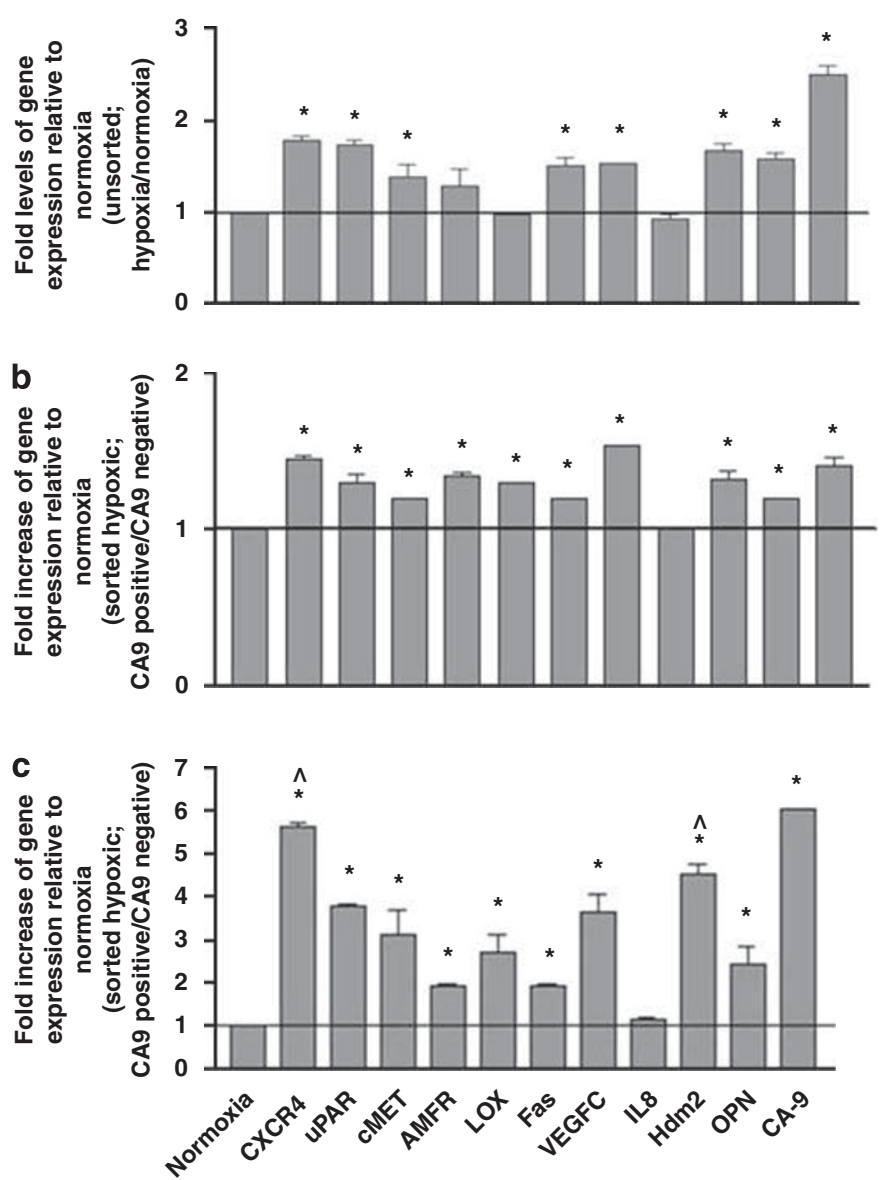
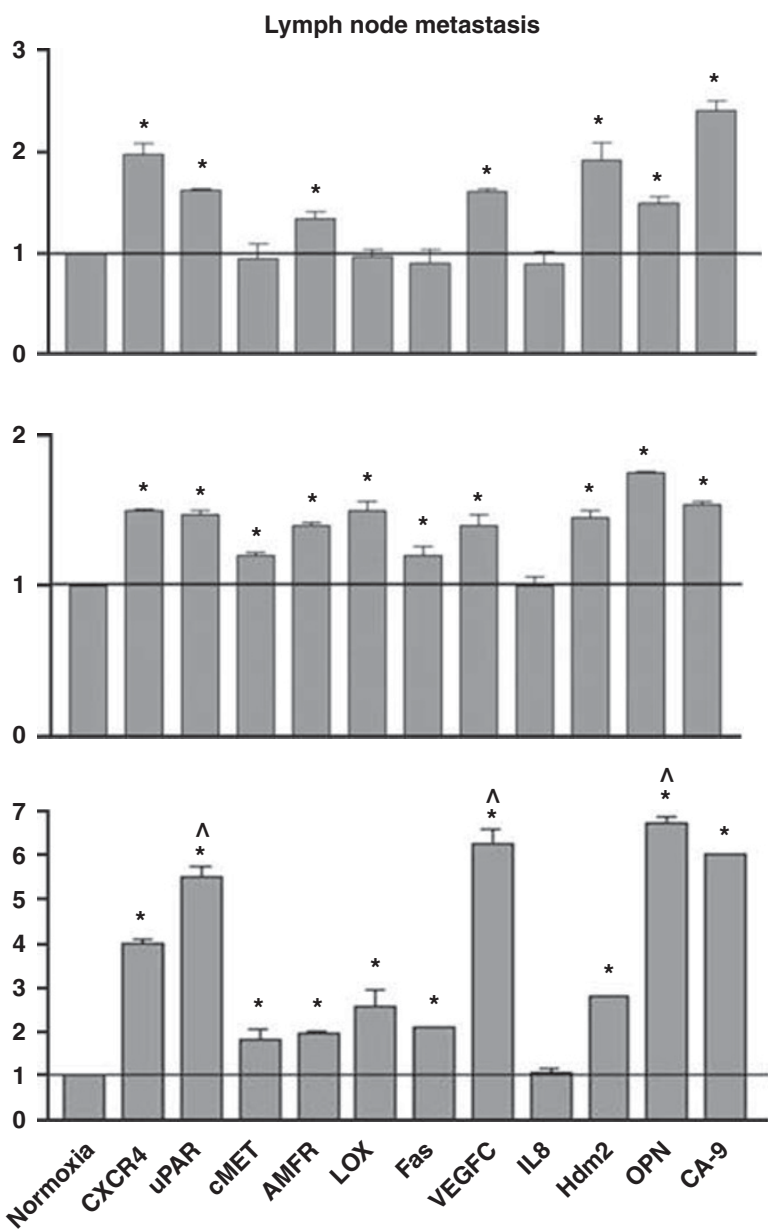

Figure 2 Gene expression changes in the sorted normoxic and hypoxic population in vivo in the orthotopic murine model of ME180 tumor cells from the primary cervix and lymph node metastatic tumors. (a) Gene expression changes in vivo in the orthotopic murine model of ME180 in unsorted (whole tumor tissue) cells from the primary cervix and lymph node metastatic tumors. Results from QRT-PCR analysis are plotted as fold increase in RNA expression from tumors after exposure to acute cyclic hypoxia ( 12 cycles- $10 \mathrm{~min} 7 \% \mathrm{O}_{2}$ bal $\mathrm{N}_{2} / 10 \mathrm{~min}$ air/4 h/day/for 3 weeks). Pooled data are shown as mean (2 ${ }^{-C_{t}}$ relative to L32) \pm s.e., $n=4$ independent experiments; ${ }^{*}$ indicates significant difference compared with normoxia (CA-9 negative); $P<0.05$. (b) Sorted normoxic population of tumor cells from the primary cervix tumors and lymph node metastatic tumors. Results from QRT-PCR analysis are plotted as fold increase in RNA expression in CA-9-positive cells relative to CA-9-negative cells. Pooled data are shown as mean \pm s.e., $n=4$ independent experiments which included $\geq 10$ mice/treatment group; ${ }^{*}$ indicates significant difference compared with normoxia (CA-9 negative); $P<0.05$. Pooled tumor-containing lymph nodes were used for each experiment. (c) Gene expression changes in the sorted hypoxic population of tumor cells from the primary cervix tumor and lymph node metastatic tumors. Results from QRT-PCR analysis are plotted as fold increase in RNA expression in CA-9-positive cells relative to CA-9-negative cells. Pooled data are shown as mean \pm s.e., $n=4$ independent experiments which included $\geq 10$ mice/treatment group; ${ }^{*}$ indicates significant difference compared with normoxia (CA-9 negative); $P<0.05$. ^Indicates significance difference between primary cervix tumors and lymph node metastasis. Refer to Materials and Methods for details. Pooled tumors-containing lymph nodes were used for each experiment.

CA-9 positivity (under hypoxia) with the target markers and VEGFC and Hmd2 showing weak-to-moderate correlation (with $<10 \%$ correlation under normoxia), which relates to the immunohistochemical analysis of these genes. These results were supported by dual-color flow cytometry analysis of tumor cells exposed to cyclic hypoxia, both in vitro and in vivo (sorted CA-9-positive cells). Figure $4 \mathrm{~b}$ and Supplementary Figure 4 also show a strong correlation of CA-9 $(>75 \%)$ with the target markers, which further supports our methodological study of using CA-9 to identify hypoxic cells in this cell-sorting technique.

\section{DISCUSSION}

Specific interest areas from tissues can be isolated by manual dissection from slides. The recent development of microdissection systems on the basis of laser technology has been useful in accelerating and extending the isolation of cell clusters or single cells with specific phenotypes or markers from tissues. In our study, we have shown the use of a simplified technique that can isolate single-cell suspensions of hypoxic cells from ME180 tumors using CA-9 as a marker. The ME180 tumors were chosen for evaluation on the basis of our earlier work showing a close correlation between CA-9 
a

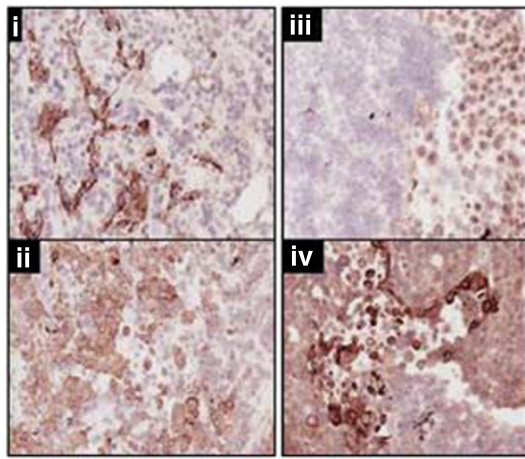

Hdm2

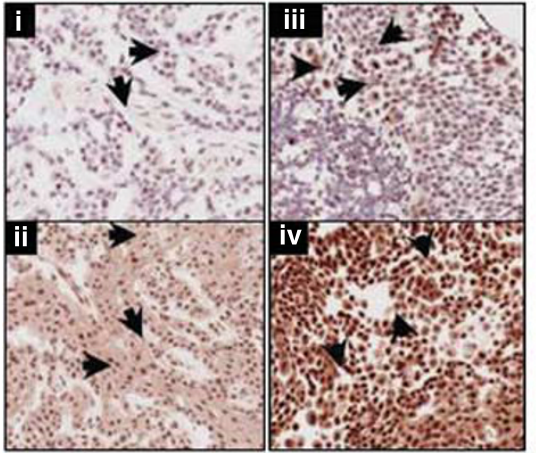

b

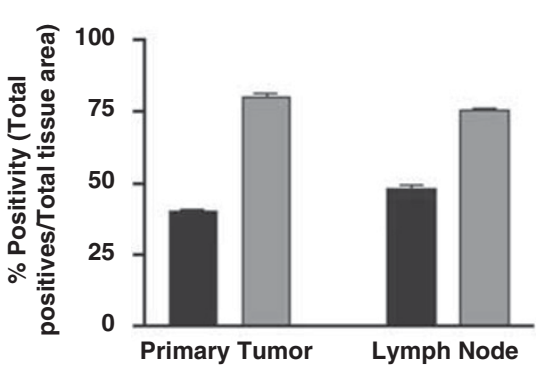

Hdm2

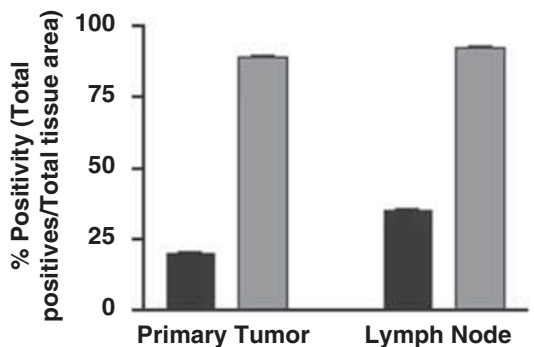

UPAR

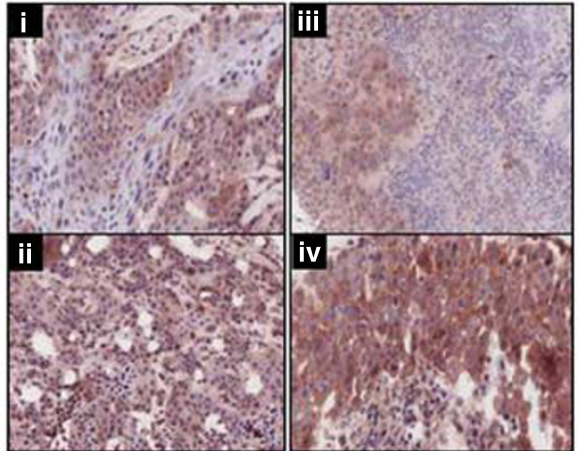

OPN

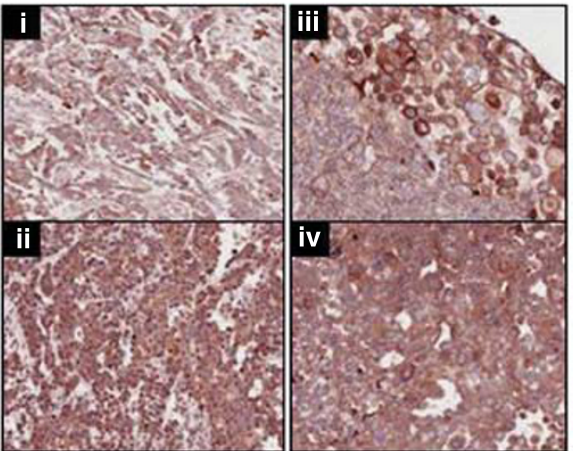

UPAR

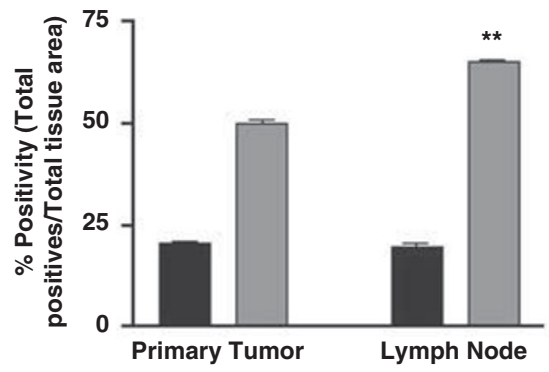

OPN

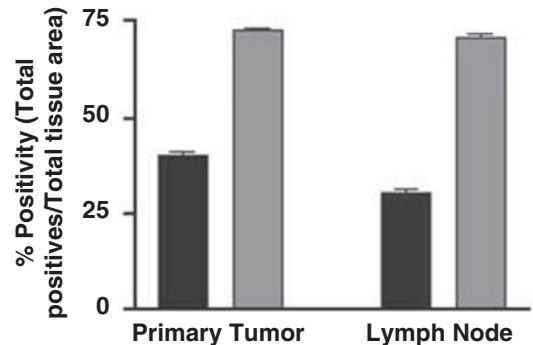

VEGFC

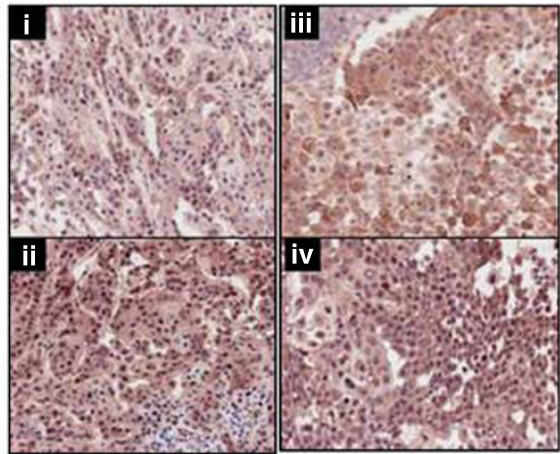

CA-9

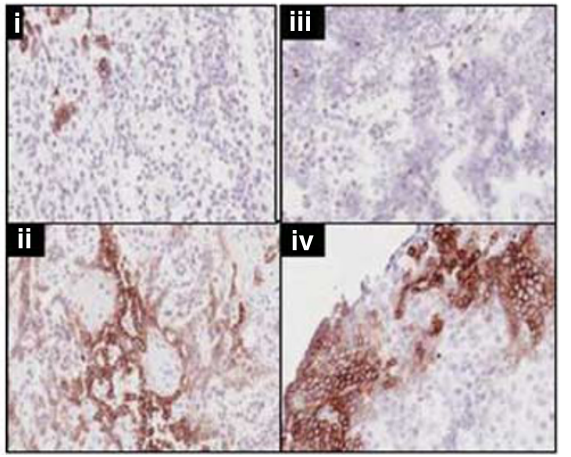

VEGFC

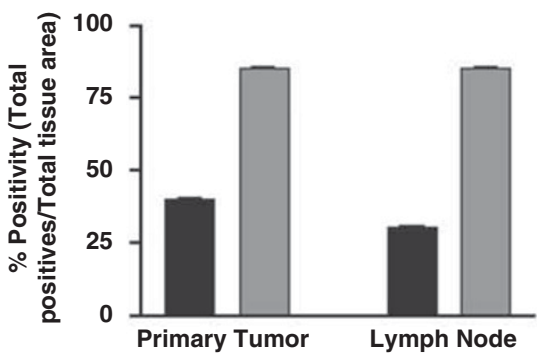

CA-9

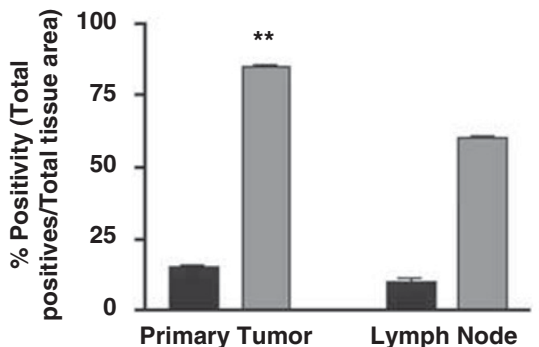

Figure 3 Immunohistochemical analysis of hypoxic-responsive and metastatic-related genes, CXCR4, uPAR, VEGFC, Hdm2, OPN, CA-9. (a) Immunostained (red) sections of tumors from the orthotopic cervical model of ME180 with hematoxylin counterstain (i) primary tumor, normoxia; (ii) primary tumor, cyclic hypoxia; (iii) lymph node metastasis, normoxia; (iv) lymph node metastasis, cyclic hypoxia. Images are representative of three independent experiments (10 mice/group). Tumors compared were obtained from same mouse/experiment. Magnification is at $20 \times$. Arrows represent nuclear staining. (b)

Quantification of immunohistochemical analysis. Positive pixel counts were used to quantify the \% positivity for each gene. \% positivity $=$ Total positive area/total tissue area (pixels). The normoxia and cyclic hypoxia groups are represented in the black and gray bars, respectively. Quantification was performed on three individual scanned images (whole section) per tumor samples to obtain a mean \pm s.e. value for each gene $(P<0.05$ between hypoxia and normoxia groups for all genes). ${ }^{*}$ Indicates significance between primary tumor and lymph node. 
a
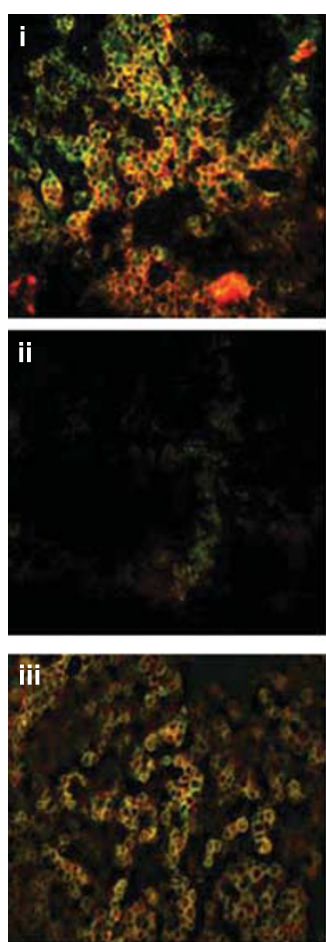

iv

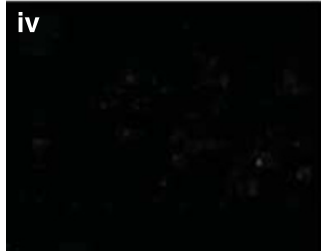

b
UPAR
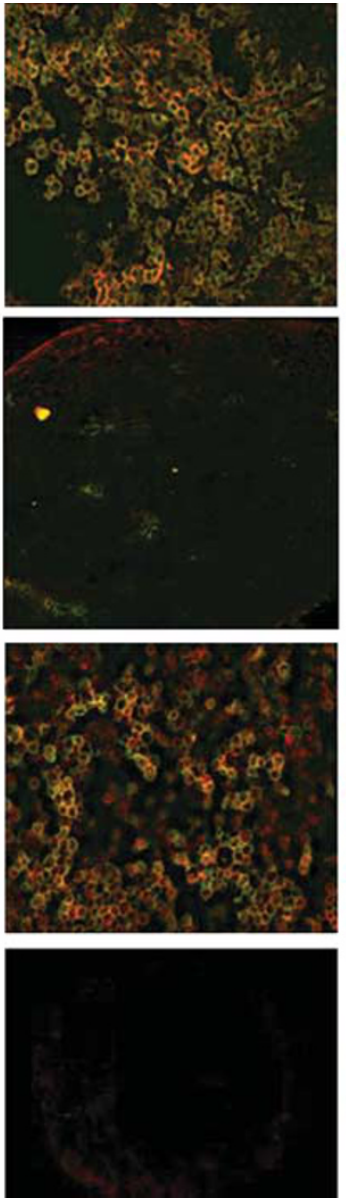

In vitro
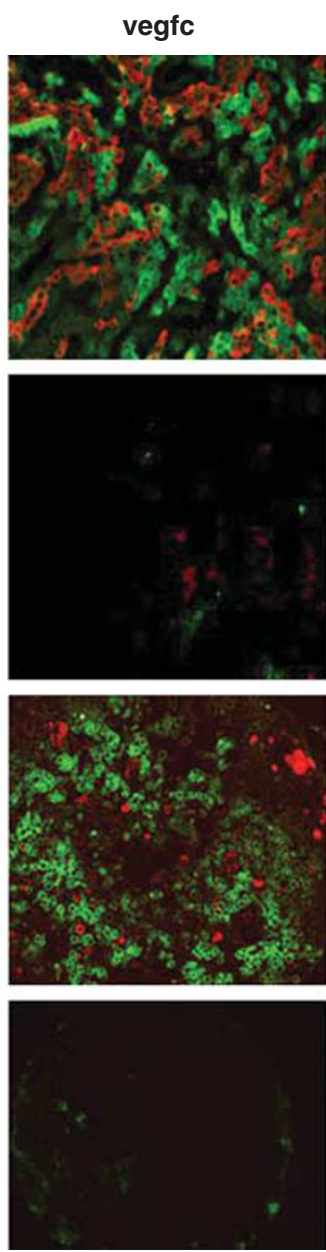

Hdm2
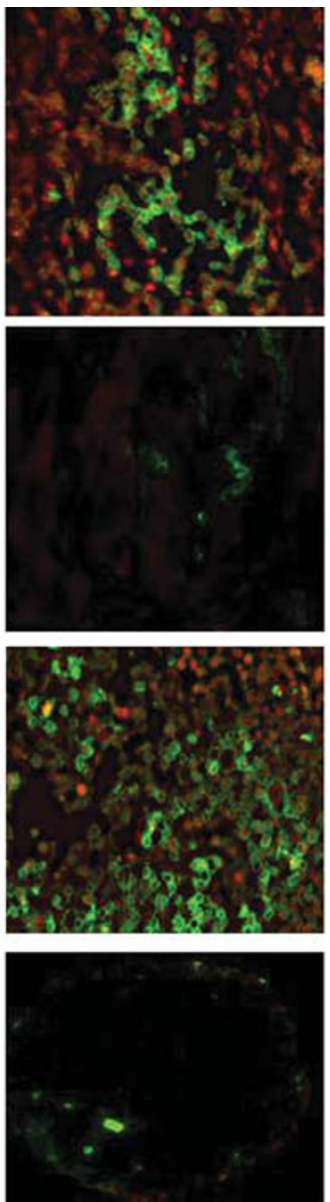

In vivo
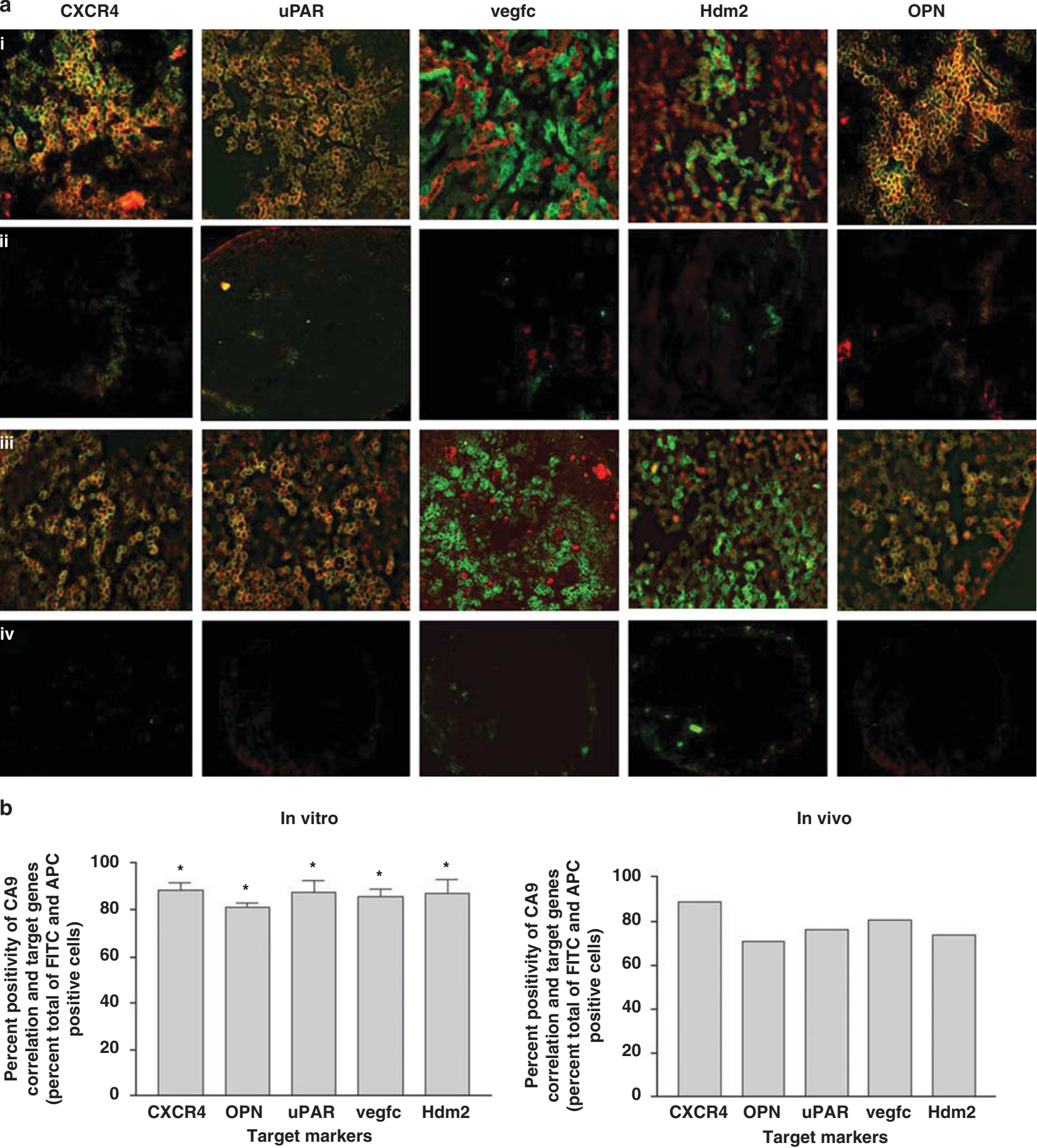

Figure 4 Correlation of target genes and CA-9 expression in tumor cells exposed to normoxia and cyclic hypoxia in vitro and in vivo.

(a) Immunofluorescence staining of primary tumor and lymph node metastasis showing CA-9 in green and the target genes represented in red in normoxia (ii) and (iv) and hypoxia (i) and (iii) treated mice from the primary tumor (i) and (ii) and lymph node metastasis (iii) and (iv). (b) Flow cytometry analysis showing the percent of CA-9 positive cells which expressed the target genes illustrated $(n=2)$ after exposure of the tumor cells to cyclic hypoxia in vitro (left panel) or of the tumors to cyclic hypoxia in vivo (CA-9 sorted cells) (right panel). ${ }^{* *} P<0.001$ between hypoxia and normoxia for all genes in vitro and in vivo.

staining and EF5 staining in these tumors. ${ }^{21}$ The sorting technique obtains cells from a volume of tissue and is hence likely to reduce the effects of heterogeneity of hypoxic regions within the tumors. We also used a tumor-specific marker
(DsRed) to select tumor cells, although this would clearly not be applicable in primary tissue specimens. However, the availability of a suitable tumor-specific marker would make this an applicable technique in primary human tumors. 
The gating procedure used was sufficient enough to ensure a large portion of DsRed (tumor positive) cells sorted from the single-cell suspension. We investigated the expression profiles of a group of metastasis-related genes in the sorted (hypoxic) population of cells from orthotopic tumors. These genes were upregulated by hypoxic exposure in vitro and most were also found to be upregulated in vivo in the primary tumors and in the lymph node metastasis in mice exposed to cyclic hypoxia. Upregulation was observed both at the mRNA and protein levels, despite the complex effects of the three-dimensional microenvironment that has been shown to have a dramatic influence on biological function and gene expression profiles. ${ }^{5,13,41}$ The majority of the studied genes is reported to be HIF-1 regulated, with the exception of OPN and Hdm2 (see Supplementary Table 3).

A novel finding of our study is the observation that the hypoxic cells sorted from tumors exposed to cyclic hypoxia showed much greater upregulation of the metastastic-related genes than those observed in the total cell population or in hypoxic cells isolated from tumors in mice exposed to normoxia (Figures 1a and b, 2a and b). The implication of these results is that the hypoxic tumor cells induced by the cyclic hypoxia regime are largely responsible for the observed gene upregulation (Figure 2c). One possible explanation of these findings may be that many of the CA-9-positive cells in tumors in mice exposed to normoxia are chronically hypoxic and have reduced transcription and translation to conserve energy. ${ }^{42,43}$ An alternative explanation might be that the CA9-positive cells in tumors from mice exposed to normoxia are not all hypoxic at the time of killing, as CA-9 is a long-lived protein and may persist for some time after reoxygenation (Figure $2 \mathrm{~b}$ and $\mathrm{c}$ ). Regardless, an important aspect of these observations of upregulation of metastasis-related genes in tumors in hypoxia-exposed mice is that they are consistent with our earlier results that exposure to hypoxia increases lymph node metastasis in this model. ${ }^{21}$

In addition to the gene expression studies, the immunolocalization analyses may be useful in part for developing new specific hypoxic markers for cervical cancer. Our localization analysis with CA-9 (hypoxia) is consistent with other reports that tumor-associated CA-9 is strongly correlated to metastases in primary cervical cancer. ${ }^{44,45}$ However, our own studies of CA-9 in primary cervix carcinomas have suggested that heterogeneity of expression in different regions of the tumors may confound the predictive value of CA-9 expression measured in biopsies from such tumors. ${ }^{36,46}$ As noted above, analysis of cell suspensions derived from biopsies may help to reduce the effect of heterogeneity. We have not addressed heterogeneity directly in this study, but it is of interest that the CA-9 positivity observed for the immunohistochemical analysis (Figure 3b) correlates in magnitude with the mean percentage of CA-9-positive cells sorted from the hypoxic and normoxic populations in both primary cervix and lymph node metastatic tumors (Figure 1). We have shown a direct relationship between hypoxia and gene expression by the tumor cells through immunofluorescence analysis and flow cytometry (Figure $4 \mathrm{a}$ and b) through staining and labeling of tumor cells with CA-9 and with the selected metastasis-related proteins. It is also of interest that the influence of the stromal interactions on gene expression is limited in the genes studied in this model, as in vitro and in vivo gene expression profiles are similar after exposure to cyclic hypoxia (data not shown).

One of the critical steps in understanding how tumors metastasize is to identify the genes involved. ${ }^{13}$ Our examination of sorted hypoxic cells revealed a gene expression profile reflecting upregulation of metastatic-related genes. Tumor models involving orthotopic transplantation are regarded as better models of human cancer on the basis of tumor histology, vascularity, gene expression, responsiveness to chemotherapy, and metastatic biology. ${ }^{47,48}$ We have used the ME180 orthotopic cervical model earlier to explore a variety of phenotypes associated with metastatic progression in relation to cervical carcinoma and lymph nodal metastasis. $^{21}$ The genes identified as responsive to acute hypoxia treatment are currently being evaluated for their involvement in metastasis in the orthotopic model using RNA interference or antibody approaches by blocking their activity or by using overexpression systems. Given their capacity to influence invasion, apoptosis, angiogenesis, migration, and survival, these genes may be important in the spread of cells to local lymph nodes, and in the establishment of secondary tumors. Our study can lead directly to the opportunity to examine therapeutic intervention, as the ME180 model has been shown to develop lymph node metastases. More specifically, this study has directed the focus on hypoxic cells from tumor cell populations to identify potential gene expression candidates for prognostic purposes. Future studies will examine these approaches in the acute-hypoxia model of ME180 cervix cancer, as well as extend the gene expression studies to biopsy specimens of human cervix cancers.

Supplementary Information accompanies the paper on the Laboratory Investigation website (http://www.laboratoryinvestigation.org)

\section{ACKNOWLEDGEMENTS}

We thank Robert Kuba for his assistance with the animal work and Dr Karstein Maseide for his assistance with tumor cell sorting and analysis. We also thank Trudey Nicklee for her assistance with the MCID program and James Ho for the immunohistochemistry and immunofluorescence work. This work was supported by a grant from the Terry Fox Foundation and the National Cancer Institute of Canada with funds raised by the Terry Fox Run.

1. Semenza GL. Hypoxia-inducible factor 1 (HIF-1) pathway. Sci STKE 2007;2007:cm8.

2. Cairns RA, Khokha R, Hill RP. Molecular mechanisms of tumor invasion and metastasis: an integrated view. Curr Mol Med 2003;3:659-671.

3. Kim JW, Gao P, Dang CV. Effects of hypoxia on tumor metabolism. Cancer Metastasis Rev 2007;26:291-298.

4. Hunter K. Host genetics influence tumor metastasis. Nat Rev Cancer 2006;6:141-146.

5. Zhou J, Schmid T, Schnitzer S, et al. Tumor hypoxia and cancer progression. Cancer Lett 2006;237:10-21. 
6. Denko NC, Fontana LA, Hudson KM, et al. Investigating hypoxic tumor physiology through gene expression patterns. Oncogene 2003:22:5907-5914.

7. Maseide K, Kalliomaki T, Hill RP. Microenvironmental effects on tumor progression and metastasis. In: Meadows GG (ed). Integration/ Interaction of Oncologic Growth. Springer: Netherlands, 2005, 1p.

8. Subarsky P, Hill RP. The hypoxic tumor microenvironment and metastatic progression. Clin Exp Metastasis 2003;20:237-250.

9. Bristow RG, Hill RP. Hypoxia and metabolism. Hypoxia, DNA repair and genetic instability. Nat Rev Cancer 2008;8:180-192.

10. Huang LE, Bindra RS, Glazer PM, et al. Hypoxia-induced genetic instability - a calculated mechanism underlying tumor progression. J Mol Med 2007;85:139-148.

11. Chan DA, Giaccia AJ. Hypoxia, gene expression, and metastasis. Cancer Metastasis Rev 2007;26:333-339.

12. Chi JT, Wang Z, Nuyten DS, et al. Gene expression programs in response to hypoxia: cell type specificity and prognostic significance in human cancers. PLoS Med 2006;3:e47.

13. Nguyen DX, Massague J. Genetic determinants of cancer metastasis. Nat Rev Genet 2007:8:341-352.

14. Laconi $\mathrm{E}$. The evolving concept of tumor microenvironments. Bioessays 2007;29:738-744.

15. Chaudary N, Hill RP. Hypoxia and metastasis. Clin Cancer Res 2007;13:1947-1949.

16. Harris AL. Hypoxia-a key regulatory factor in tumor growth. Nat Rev Cancer 2002;2:38-47.

17. Prabhakar NR, Kumar GK, Nanduri J, et al. ROS signaling in systemic and cellular responses to chronic intermittent hypoxia. Antioxid Redox Signal 2007;9:1397-1403.

18. Semenza GL, Prabhakar NR. HIF-1-dependent respiratory, cardiovascular, and redox responses to chronic intermittent hypoxia. Antioxid Redox Signal 2007;9:1391-1396.

19. Pitson G, Fyles $A$, Milosevic $M$, et al. Tumor size and oxygenation are independent predictors of nodal diseases in patients with cervix cancer. Int J Radiat Oncol Biol Phys 2001;51:699-703.

20. Fyles A, Milosevic M, Hedley D, et al. Tumor hypoxia has independent predictor impact only in patients with node-negative cervix cancer. J Clin Oncol 2002;20:680-687.

21. Cairns RA, Hill RP. Acute hypoxia enhances spontaneous lymph node metastasis in an orthotopic murine model of human cervical carcinoma. Cancer Res 2004;64:2054-2061.

22. Brizel DM, Rosner GL, Prosnitz LR, et al. Patterns and variability of tumor oxygenation in human soft tissue sarcomas, cervical carcinomas, and lymph node metastases. Int J Radiat Oncol Biol Phys 1995;32:1121-1125.

23. Bachtiary B, Boutros PC, Pintilie $M$, et al. Gene expression profiling in cervical cancer: an exploration of intratumor heterogeneity. Clin Cancer Res 2006;12:5632-5640.

24. Rofstad EK, Galappathi K, Mathiesen B, et al. Fluctuating and diffusionlimited hypoxia in hypoxia-induced metastasis. Clin Cancer Res 2007;13:1971-1978.

25. Fend $F$, Raffeld M. Laser capture microdissection in pathology. J Clin Pathol 2000;53:666-672.

26. Mueller MM, Fusenig NE. Friends or foes-bipolar effects of the tumor stroma in cancer. Nat Rev Cancer 2004:4:839-849.

27. Olive PL, Aquino-Parsons C, MacPhail SH, et al. Carbonic anhydrase 9 as an endogenous marker for hypoxic cells in cervical cancer. Cancer Res 2001;61:8924-8929.

28. Allalunis-Turner MJ, Siemann DW. Recovery of cell subpopulations from human tumor xenografts following dissociation with different enzymes. Br J Cancer 1986;54:615-622.
29. Keng PC, Allalunis-Turner J, Siemann DW. Evaluation of cell subpopulations isolated from human tumor xenografts by centrifugal elutriation. Int J Radiat Oncol Biol Phys 1990;18:1061-1067.

30. Bennewith $\mathrm{KL}$, Durand RE. Quantifying transient hypoxia in human tumor xenografts by flow cytometry. Cancer Res 2004;64:6183-6189.

31. Vordermark D, Kaffer A, Riedl S, et al. Characterization of carbonic anhydrase IX (CA IX) as an endogenous marker of chronic hypoxia in live human tumor cells. Int J Radiat Oncol Biol Phys 2005;61: 1197-1207.

32. Cairns RA, Hill RP. A fluorescent orthotopic model of metastatic cervical carcinoma. Clin Exp Metastasis 2004;21:275-281.

33. Maseide K, Kandel RA, Bell RS, et al. Carbonic anhydrase IX as a marker for poor prognosis in soft tissue sarcoma. Clin Cancer Res 2004;10:4464-4471.

34. Swietach $\mathrm{P}$, Vaughan-Jones RD, Harris AL. Regulation of tumor $\mathrm{pH}$ and the role of carbonic anhydrase 9. Cancer Metastasis Rev 2007;26:299-310.

35. Wykoff CC, Beasley NJ, Watson PH, et al. Hypoxia-inducible expression of tumor-associated carbonic anhydrases. Cancer Res 2000;60: 7075-7083.

36. lakovlev VV, Pintilie M, Morrison A, et al. Effect of distributional heterogeneity on the analysis of tumor hypoxia based on carbonic anhydrase IX. Lab Invest 2007;87:1206-1217.

37. Shim H, Lau SK, Devi S, et al. Lower expression of CXCR4 in lymph node metastases than in primary breast cancers: potential regulation by ligand-dependent degradation and HIF-1alpha. Biochem Biophys Res Commun 2006;346:252-258.

38. Ueda $M$, Hung YC, Terai $Y$, et al. Vascular endothelial growth factor- $C$ expression and invasive phenotype in ovarian carcinomas. Clin Cancer Res 2005;11:3225-3232.

39. Van Trappen PO, Steele D, Lowe DG, et al. Expression of vascular endothelial growth factor (VEGF)-C and VEGF-D, and their receptor VEGFR-3, during different stages of cervical carcinogenesis. J Pathol 2003;201:544-554

40. Memarzadeh S, Kozak KR, Chang L, et al. Urokinase plasminogen activator receptor: prognostic biomarker for endometrial cancer. Proc Natl Acad Sci USA 2002;99:10647-10652.

41. Wittekind C, Neid M. Cancer invasion and metastasis. Oncology 2005;69(Suppl 1):14-16.

42. Koritzinsky M, Magagnin MG, van den Beucken T, et al. Gene expression during acute and prolonged hypoxia is regulated by distinct mechanisms of translational control. EMBO J 2006;25: 1114-1125.

43. Wouters BG, van den Beucken T, Magagnin MG, et al. Control of the hypoxic response through regulation of mRNA translation. Semin Cell Dev Biol 2005;16:487-501.

44. Kim JY, Shin HJ, Kim TH, et al. Tumor-associated carbonic anhydrases are linked to metastases in primary cervical cancer. J Cancer Res Clin Oncol 2006;132:302-308.

45. Loncaster JA, Harris AL, Davidson $\mathrm{SE}_{\text {, }}$ et al. Carbonic anhydrase (CA IX) expression, a potential new intrinsic marker of hypoxia: correlations with tumor oxygen measurements and prognosis in locally advanced carcinoma of the cervix. Cancer Res 2001;61:6394-6399.

46. Hedley D, Pintilie M, Woo J, et al. Carbonic anhydrase IX expression, hypoxia, and prognosis in patients with uterine cervical carcinomas. Clin Cancer Res 2003;9:5666-5674.

47. Khanna C, Prehn J, Yeung C, et al. An orthotopic model of murine osteosarcoma with clonally related variants differing in pulmonary metastatic potential. Clin Exp Metastasis 2000;18:261-271.

48. Khanna C, Hunter K. Modeling metastasis in vivo. Carcinogenesis 2005;26:513-523. 